\title{
Un diagnostic échographique en un clin d'œil
}

\section{A Diagnosis at a Glance}

\section{Marchetti · J. Espenan - G. Vesselle}

Reçu le 17 mai 2018; accepté le 5 juillet 2018

(C) SFMU et Lavoisier SAS 2018

Un patient de 18 ans se présente aux urgences pour une baisse de l'acuité visuelle de l'œil droit depuis une semaine, associée à l'apparition d'un voile noir amputant une partie du champ visuel de l'œil droit. Il n'a pas d'antécédent particulier. Son œil est blanc et indolore. L'examen ophtalmologique aux urgences confirme la baisse d'acuité visuelle et une amputation significative du champ visuel. Il n'existe ni diplopie ni trouble de l'oculomotricité. L'examen de l'œil gauche est sans particularité, de même que l'examen neurologique. Une échographie oculaire comparative des deux yeux est réalisée par le médecin urgentiste à l'aide d'une sonde haute fréquence de $12 \mathrm{Mhz}$ et un programme d'étude vasculaire (appareil VIVID S5, General Electric, Boston, États-Unis). L'examen est réalisé avec les paupières fermées. Il révèle une structure isoéchogène finement hétérogène, semblant appendue au bord nasal du segment postérieur. S'y associe au contact un aspect hétérogène limité par une fine membrane hyperéchogène flottante de la partie postérieure du vitré, faisant suspecter un décollement du vitré, ou de rétine, associé. Le patient est hospitalisé dans le service d'ophtalmologie et bénéficie au cours de sa prise en charge d'une IRM afin d'étayer le diagnostic (Fig. 1). Ces examens confirment la présence d'un décollement de rétine associé à une masse tissulaire centimétrique, dont les caractéristiques (notamment par le signal des pigments mélaniques) évoquent un mélanome choroïdien. Un traitement chirurgical, consistant en l'exérèse de la lésion, a été réalisé

M. Marchetti $(\bowtie)$

Pôle de médecine d'urgence, CHU de Poitiers,

2, rue de la Milétrie, F-86000 Poitiers, France

e-mail : matthieu.marchetti@chu-poitiers.fr

J. Espenan

Service d'ophtalmologie, CHU de Poitiers,

2, rue de la Milétrie, F-86000 Poitiers, France

G. Vesselle

Service d'imagerie diagnostique,

fonctionnelle et interventionnelle,

CHU de Poitiers, 2, rue de la Milétrie,

F-86000 Poitiers, France

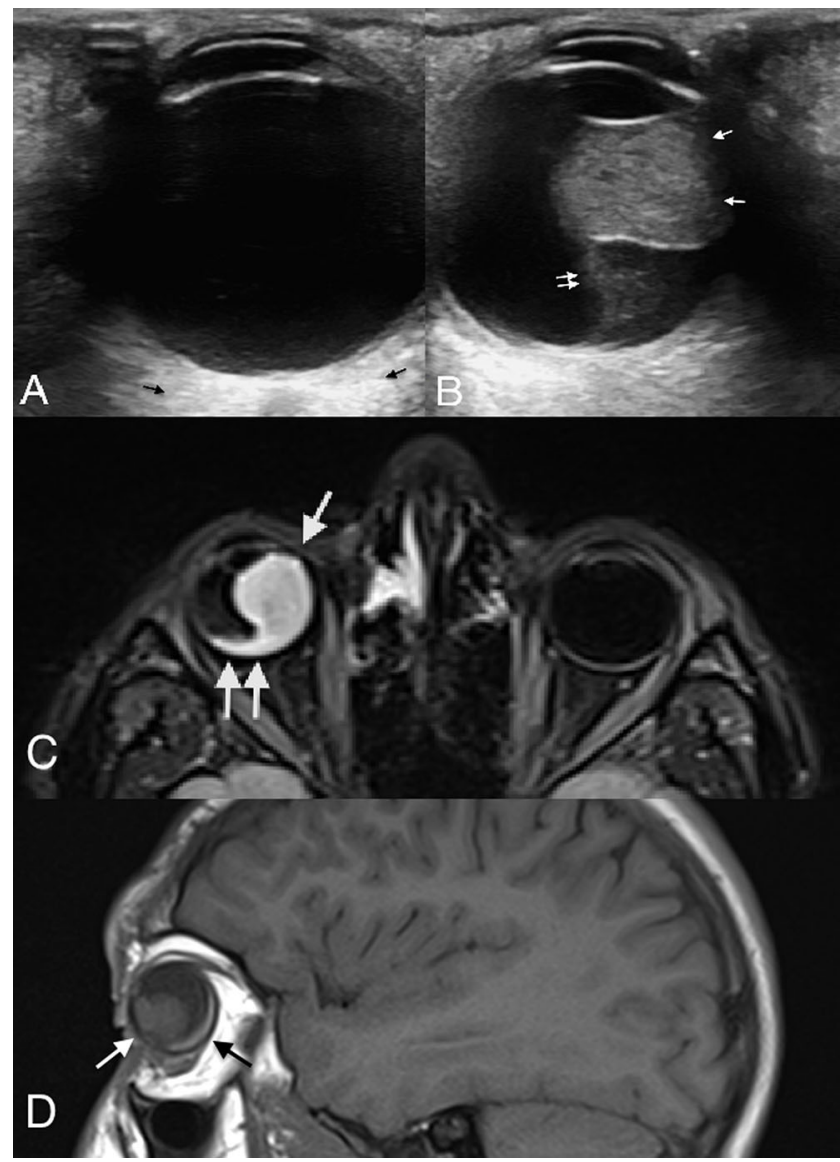

Fig. 1 A. Échographie de l'œil gauche en mode B. Eil calme avec contenu liquidien hypoéchogène homogène et renforcement postérieur (flèches noires). B. Échographie de l'œil droit en mode B. Syndrome de masse hyperéchogène discrètement hétérogène du versant médial du segment postérieur du globe oculaire (flèches blanches) et composante postérieure globalement hétérogène hypoéchogène limitée par une membrane mobile lors de l'échographie (double flèche). C. IRM correspondante en séquence axiale Flair retrouvant des anomalies similaires sur la lésion du versant médial (flèche) et le décollement postérieur (double flèche). D. Séquence T1 en coupe sagittale. Syndrome de masse (flèche blanche) et décollement de rétine (flèche noire) 
secondairement. L'analyse anatomopathologique a confirmé le diagnostic. Les services d'urgences sont confrontés à une part non négligeable de patients consultant pour des motifs ophtalmologiques [1]. Le médecin urgentiste tient un rôle déterminant dans l'orientation de ces patients, notamment dans les centres ne disposant pas de service d'ophtalmologie. L'échographie oculaire ne fait pas encore partie du champ de compétence du médecin urgentiste. Il serait nécessaire de réaliser des études sur le sujet afin d'en évaluer la pertinence diagnostique et la courbe d'apprentissage par l'urgentiste. Par ailleurs, il faut rappeler que l'échographie oculaire doit être effectuée avec un appareil respectant certaines contraintes : index mécanique inférieur à 0,23 et un index thermique inférieur à 1 . Pour les pathologies intéressant la chambre antérieure de l'œil, l'examen à l'ophtalmoscope direct reste facilement accessible dans la plupart des services d'urgences. Les mélanomes du corps ciliaire et de la choroïde sont les tumeurs les plus fréquemment symptomatiques de l'adulte [2].

\section{Références}

1. Jeannin A, Mouriaux F, Mortemousque B (2016) Prise en charge des urgences ophtalmologiques en structure des urgences : étude multicentrique rétrospective. J Fr Ophtalmol 39:589-95

2. Zografos L (2002) Tumeurs intraoculaires. Elsevier-Masson Éditions, Paris 